\title{
A FULLY-PASSIVE WIRELESS MICROFABRICATED NEURO-RECORDER
}

\author{
Helen N. Schwerdt ${ }^{1}$, Wencheng $X u^{l}$, Sameer Shekhar ${ }^{1}$, \\ Abbas Abbaspour-Tamijani ${ }^{1}$, Bruce C. Towe ${ }^{2}$, and Junseok Chae ${ }^{I}$ \\ ${ }^{1}$ School of Electrical, Computer, and Energy Engineering, Arizona State University, Tempe, USA \\ ${ }^{2}$ School of Biological and Health Systems Engineering, Arizona State University, Tempe, USA
}

\begin{abstract}
This paper reports a completely passive and wireless MEMS device for potential use in recording neuro-potentials in-vivo. Its operating principle utilizes an implantable circuit or "tag" to backscatter data to an external interrogator that supplies the fundamental carrier. The backscattering circuit relies solely on its nonlinear components, varactor diodes, which mix and backscatter neuro-potentials with the supplied carrier. Performance of the MEMS neuro-recorder was demonstrated using emulated and in-vivo neuro-potentials as low as $500 \mu \mathrm{V}_{\mathrm{P}-\mathrm{P}}$ and up to $3 \mathrm{kHz}$.
\end{abstract}

\section{INTRODUCTION}

Current wireless neuro-recording microsystems employ multi-component packages comprised of sophisticated circuitry located between the scalp and skull, which is then wired to micromachined electrode arrays implanted into the cortex of the brain [1-2]. In order to remove these interconnects and improve signal acquisition quality some have monolithically integrated the circuits onto the electrodes and/or fabricated die-level post-CMOS electrodes on-chip [3]. These advancements make it possible to monitor in-vivo potentials with extremely high spatial and temporal resolution. Nevertheless, challenges remain due to the complexity of wireless telemetry. To bypass some of this complexity, fully-passive techniques have been previously implemented in meso-scale systems [4-5]. In this paper, we present an alternative, significantly less complex microsystem for passive and wireless neuro-recording consisting of only two varactors, three MIM capacitors, and an on-chip planar antenna.

\section{DEVICE OPERATION AND FABRICATION}

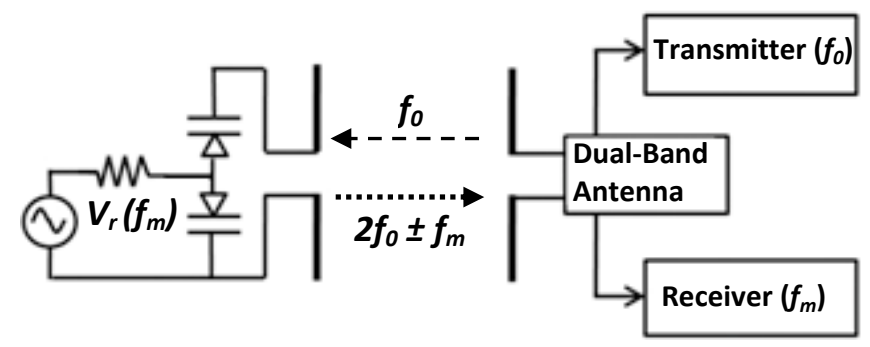

Figure 1: A simplified schematic of the fully-passive and wireless neuro-recorder. $V_{r}\left(f_{m}\right)$ is the low frequency neuro-potential signal, $f_{0}$ is the carrier, and $2 f_{0} \pm f_{m}$ is the backscattered modulated signal.

The basic passive and wireless operation is illustrated in Fig. 1. An RF excitation carrier $\left(f_{0}\right)$ generated by an external interrogator mixes neuro-potentials $\left(f_{m}\right)$ through nonlinear varactors, backscattering third-order intermodulation products $2 f_{0} \pm f_{m}$. An illustration of the neuro-recorder is shown in Fig. 2a. The fabrication process uses seven masks (Fig. 2b). KOH-etched trenches on the front and back form the sockets for the varactors and probe feedthroughs, respectively. The frontside consists of a planar antenna, MIM capacitors, and varactors, and the backside is patterned with through-wafer interconnects to interface with neuro-potentials (Fig. 3).

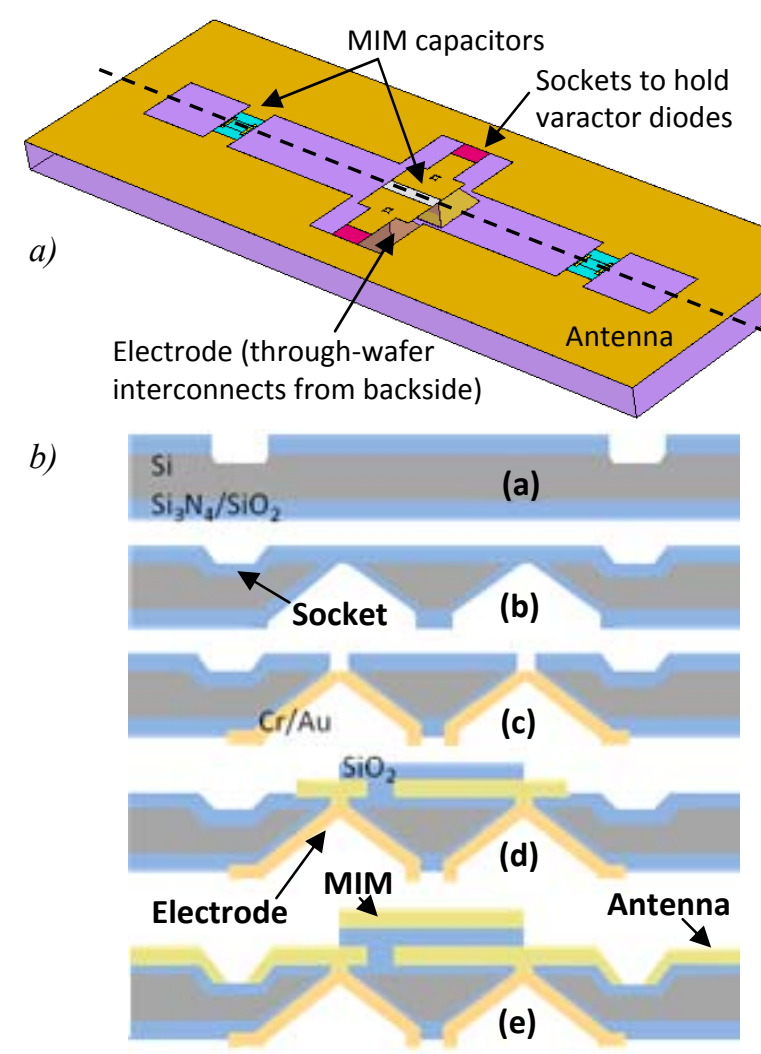

Figure 2: a) An oblique view of the MEMS neuro-recorder with on-chip components and cross-sectional axis labeled (dashed line). b) Cross-sectional fabrication process flow: (a) pattering of $\mathrm{Si} / \mathrm{Si}_{3} \mathrm{~N}_{4} / \mathrm{SiO}_{2}$ on front, (b) backside $\mathrm{KOH}$ etching for through-wafer interconnects and PECVD of $\mathrm{SiO}_{2}$ for insulation, (c) depositing $\mathrm{Cr} / \mathrm{Au}$, and defining vias to electrodes on front, (d) depositing $\mathrm{Cr} / \mathrm{Au}$ for bottom MIM plate and via interconnect, and PECVD of $\mathrm{SiO}_{2}$ for MIM oxide layer, (e) depositing Cr/Au for antenna, MIM, and pads for diodes.

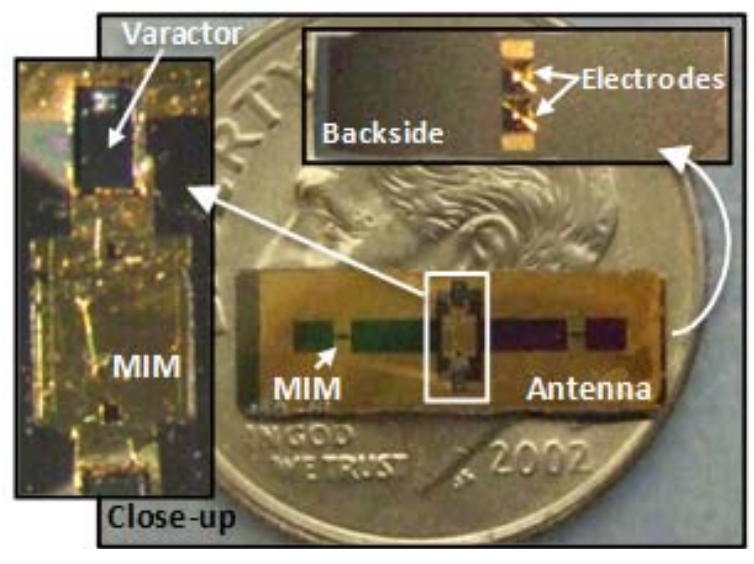

Figure 3: Photograph of a fabricated MEMS neuro-recorder placed above a dime (topside) with backside and close-up views. 


\section{TESTING}

The MEMS neuro-recorder was characterized using i) emulated neuro-potentials and ii) in-vivo compound action potentials (CAPs) from a frog's sciatic nerve. A phase-synchronous demodulator was built to retrieve the wirelessly transmitted signals. The frog's nerve was stimulated by pulsing current into the lower end of the spinal column generating CAPs in the range of 10-1000 $\mu \mathrm{V}$ down the nerve bundle. The CAPs acquired with the MEMS recorder ranged from $2-140 \mathrm{~Hz}$ and $500-1000 \mu \mathrm{V}$.

\section{RESULTS}

The passive wireless telemetry operates at 2.2-2.45/4.4-4.9 $\mathrm{GHz}\left(f_{0} / 2 f_{0}\right)$ for emulated neuro-potentials up to $3 \mathrm{kHz}\left(f_{m}\right)$ and as low as $500 \mu \mathrm{V}$. Fig. 4 shows the measured frequency spectrum where a sinusoidal input signal generated by a function generator, of $3.4 \mathrm{mV}$ at $400 \mathrm{~Hz}\left(f_{m}\right)$ is mixed with the backscattered carrier $\left(2 f_{0}\right.$, $4.41 \mathrm{GHz}$ ) resulting in the observed sidebands at $15 \mathrm{~dB}$ above the noise floor. Table 1 summarizes the measured specifications of the MEMS neuro-recording device. Fig. 5 shows CAPs from the frog as detected from the MEMS device and from measurements using the reference electrode.

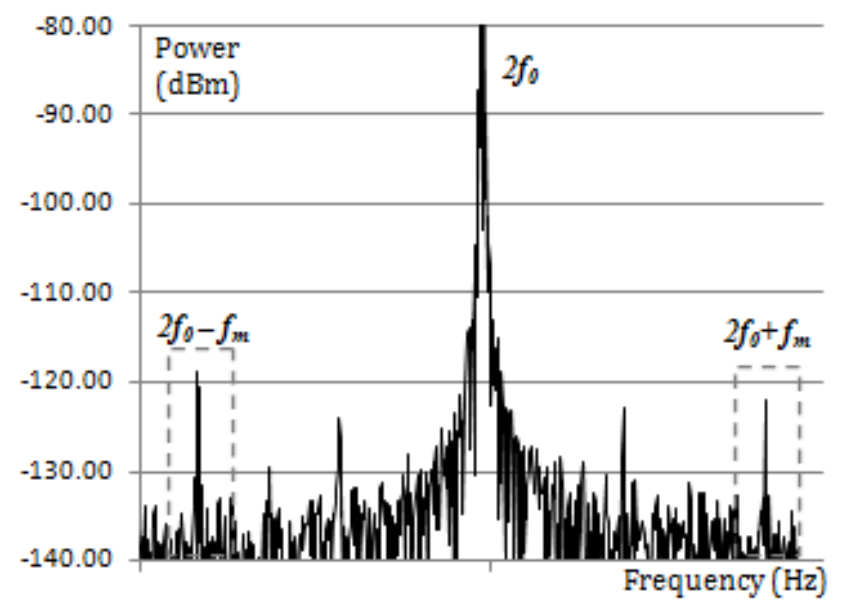

Figure 4: Measured spectrum for $3.4 \mathrm{mV}\left(f_{m}=400 \mathrm{~Hz}\right)$ sinusoidal input and $14.8 \mathrm{dBm}$ radiated from the source. Center backscattered frequency (2fo) shown at $4.409999220 \mathrm{GHz}$ with $400 \mathrm{~Hz}$ offset sidebands approximately $15 \mathrm{~dB}$ above the noisefloor (boxed in).

Table 1: Summary of specifications for MEMS device.

\begin{tabular}{|l|l|l|}
\hline Size & & $12 \times 4 \times 0.5 \mathrm{~mm}^{3}$ \\
\hline Detected Input & Bandwidth & $5-3000 \mathrm{~Hz}$ \\
Signals & Amplitude & $\geq 500 \mathrm{uV}$ \\
& Distance & $\leq 2.5 \mathrm{~cm}$ \\
\hline RF Transmission & Radiated Power & $0-18 \mathrm{dBm}$ \\
& Frequency & $2.2-2.45 \mathrm{GHz}$ \\
\hline RF Reception & Sideband Level & $\leq-105 \mathrm{dBm}$ \\
& Noise Floor & $\approx-134 \mathrm{dBm}$ \\
& Frequency & $4.4-4.9 \mathrm{GHz}$ \\
\hline
\end{tabular}

\section{DISCUSSION}

At this stage, the MEMS neuro-recorder may be suitable for detecting signals in the peripheral nervous system (PNS), especially in motor nerve bundles where CAPs reach up to hundreds of microvolts to several millivolts. The CAP measurements (Fig. 5) suggest the neuro-recorder may be capable of detecting neurological signals as low as $500 \mu \mathrm{V}$. Below $500 \mu \mathrm{V}$, the current system becomes dominated by noise, including $60 \mathrm{~Hz}$ noise. The frequency of the noise may coincide with the frequency of the neuro-potentials and can completely screen out the targeted CAPs. However, because the current limitations in minimum detectable signal may result from external noise sources, future work to diminish noise may improve the device's sensitivity and enable signal detection on the same order as cortical potentials.

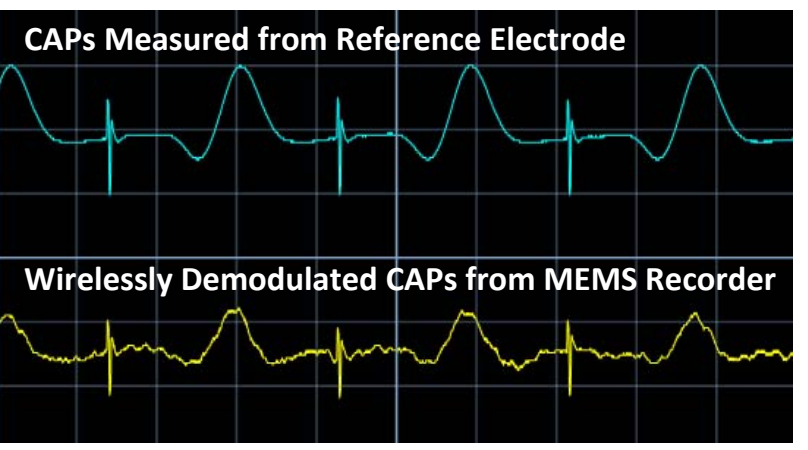

Figure 5: CAPs generated from a frog measured by a reference electrode (top, $0.1 \mathrm{~V} / \mathrm{div}, 0.0025 \mathrm{~s} /$ div) at $500 \mu V_{p-p}$ and wirelessly acquired from the MEMS neuro-recorder (bottom, $0.5 \mathrm{~V} / d i v, 0.0025$ s/div). The sharp spikes represent artifacts of the pulsed current stimulation, which also travel down the nerve. The oscilloscope used 128 point averaging to remove some of the background noise.

\section{ACKNOWLEDGEMENT}

The authors thank Mr. Jaeyoong Cho for depositing PECVD films, and Patrick Larson for his assistance with frog testing. This work is partially supported by NSF (ECCS-0702227), NIH (5R21NS059815-02), and NASA-GRC fellowship (NNX09AK93H).

\section{REFERENCES}

[1] A.M. Sodagar, G.E. Perlin, Y. Yao, K. Najafi, K. D. Wise, “An Implantable 64-Channel Wireless Microsystem for Single-Unit Neural Recording," IEEE J. Solid-State Circuits, 44 (9), 2591-2604, 2009.

[2] Y.K. Song, D.A. Borton, S. Park, W.R. Patterson, et. al., "Active Microelectronic Neurosensor Arrays for Implantable Brain Communication Interfaces," IEEE Trans. Neural Sys. Rehab. Eng., 17 (4), 339-345, 2009.

[3] M. Ho, H. Chen, F. Tseng, S. Yeh, M. S. Lu, "CMOS Micromachined Probes by Die-Level Fabrication for Extracellular Neural Recording," J. Micromech. Microeng., 17, 283-290, 2007.

[4] B.C. Towe, "Passive Backscatter Biotelemetry for Neural Interfacing," Proc. IEEE Int. EMBS Conf. Neural Engineering, 144-147, 2007.

[5] A. Abbaspour-Tamijani, M. Farooqui, B. Towe, and J. Chae, "A Miniature Fully-passive Microwave Back-scattering Device for Short-range Telemetry of Neural Potentials," IEEE EMBS, Vancouver, Canada, 2008.

\section{CONTACT}

H.N. Schwerdt, hschwerd@asu.edu 